\title{
REVIEW ON OPTIMIZATION TECHNIQUES USED FOR IMAGE COMPRESSION
}

\author{
Shet Reshma Prakash ${ }^{1}$, Vrinda Shetty ${ }^{2}$ \\ ${ }^{1}$ Student, Computer Science \& Engineering, SVIT, Karnataka, India \\ ${ }^{2}$ Asst. Professor \& HOD, Information Science \& Engineering, SVIT, Karnataka, India
}

\begin{abstract}
Image compression is most essential requirement for efficient utilization of storage space and transmission bandwidth. Image compression technique involves reducing the size of the image without degrading the quality of the image. Currently many image compression algorithms are used to deal with increasing amount of data involved but still finding the alternative solution is the area of research. This paper reviews some of the Meta heuristic optimization algorithms used for image compression. These algorithms are based on swarm intelligence. Swarm intelligence is a relatively new area that deals with the study of behavior among many entities or objects interacting within the natural or artificial systems. In past few years Swarm Intelligence based algorithms have been applied to a wide variety of problems in combinatorial and continuous optimization, telecommunications, swarm robotics, networking, image processing etc. This paper provides an insight of many optimization techniques used for image compression like Ant Colony Optimization (ACO) algorithm, Harmony Search Algorithm (HSA) and Artificial Bee Colony algorithm, Particle Swarm Optimization (PSO) and Genetic Algorithm (GA). Ant Colony Optimization algorithm is inspired by the behavior among real ant's while searching for the food source. Harmony Search Algorithm is inspired by the harmony improvisation process followed while playing music. Particle swarm optimization is an optimization technique inspired by social behavior of bird flocking or fish schooling. Artificial Bee Colony algorithm is motivated by the behavior exhibited by honey bees while searching for the food source. Genetic Algorithm is based on processes observed in the natural evolution.
\end{abstract}

Keywords: Image compression, Ant Colony Optimization (ACO), Harmony Search Algorithm (HAS), Artificial Bee Colony (ABC) algorithm, Particle Swarm Optimization (PSO), Genetic Algorithm (GA).

\section{INTRODUCTION}

Data compression is beneficial because it reduces the consumption of expensive resources like bandwidth and hard disk space. Image Compression is a technique which is concerned with reducing the number of bits required to store and transmit the image without appreciable loss of information. There are two types of image compression namely lossy and lossless. In lossy technique there is loss of information and hence it is not possible to exactly recover the original image. Also the quality of the image gets degraded and compression ratio is high. In lossless technique no information is lost and hence the original image can be exactly reconstructed but the compression ratio is low.

Image compression is most essential solution when the images are transmitted over the internet or downloaded from the web pages. There are various techniques for image compression. JPEG is the most commonly used lossy image compression technique based on Discrete Cosine Transform (DCT). JPEG 2000 standard uses Discrete Wavelet Transform (DWT) which decomposes the image into four frequency sub bands i.e. LL, LH, HL and HH. In the past few years many Meta-heuristic Optimization algorithms have been used to compress the images with minimal loss of information. There are three types of Meta-heuristic Optimization algorithms i.e. Physics based, Swarm
Intelligence based and Evolutionary based. Ant Colony Optimization (ACO), Harmony Search Algorithm (HSA) and Artificial Bee Colony (ABC) algorithm and Particle Swarm Optimization (PSO) are swarm intelligence based Meta-heuristic Optimization algorithms and only Genetic Algorithm is Evolution based Meta-heuristic algorithm. One important advantage of using the Optimization technique is that is helps to increase the compression ratio while maintaining good quality for the image.

Shu-Chuan Chu et. al [1] and R. C. Eberhert et. al [2] have discussed about various Swarm Intelligence based Optimization Algorithms. K. Uma et. al [3] have given the overview of various fractal image compression algorithms. Harsha D. Zope et. al [4] have done comparative study for various image compression techniques like Discrete Cosine Transform(DCT),Discrete Wavelet Transform(DWT) and Wavelet Packet Decomposition(WPD). Dervis Karaboga et. al [5] has discussed the Artificial Bee Colony(ABC) algorithm in detail. Ryan Rey M. Daga et. al [6], Sarang Bansod et.al [7] and Z. W. Geem et. al [8] have provided detailed information on Harmony Search Algorithm(HSA) and its applications. Simranjeet Kaur et.al [9] have proposed ACO based algorithm for solving the problems like image edge detection, image compression and image segmentation. Christian Blum [10] and C. Martinez [11] have discussed the use of Ant Colony Optimization (ACO) algorithm for image compression. M. Mohamed Ismail et.al [12][13] have 
proposed Hybrid Lifting and Adaptive Lifting based Image Compression using Artificial Bee Colony(ABC) algorithms. Mitra et. al [14] and Chen Shuwang An Tao Hao Litao [15] have discussed image compression using Genetic Algorithm(GA).

The paper is organized as follows. Section 2, 3, 4, 5, 6 provides detailed information of Ant Colony Optimization (ACO), Harmony Search Algorithm (HSA), Artificial Bee Colony (ABC) algorithm, Particle Swarm Optimization (PSO) and Genetic Algorithm (GA). Section 7 and beyond gives the conclusion and references.

\section{ANT COLONY OPTIMIZATION}

Ant Colony Optimization(ACO) algorithm was introduced by Marco Dorigo in 1992 to generate the appropriate solutions for discrete optimization problems, continuous optimization problems and some real world problems like Travelling Salesman problem, routing and load balancing. It mimics the foraging behavior of real ant colonies. There are many variants of ACO namely Ant System, Elitist Ant Colony System, Max-Min Ant System, Rank Based Ant System etc.

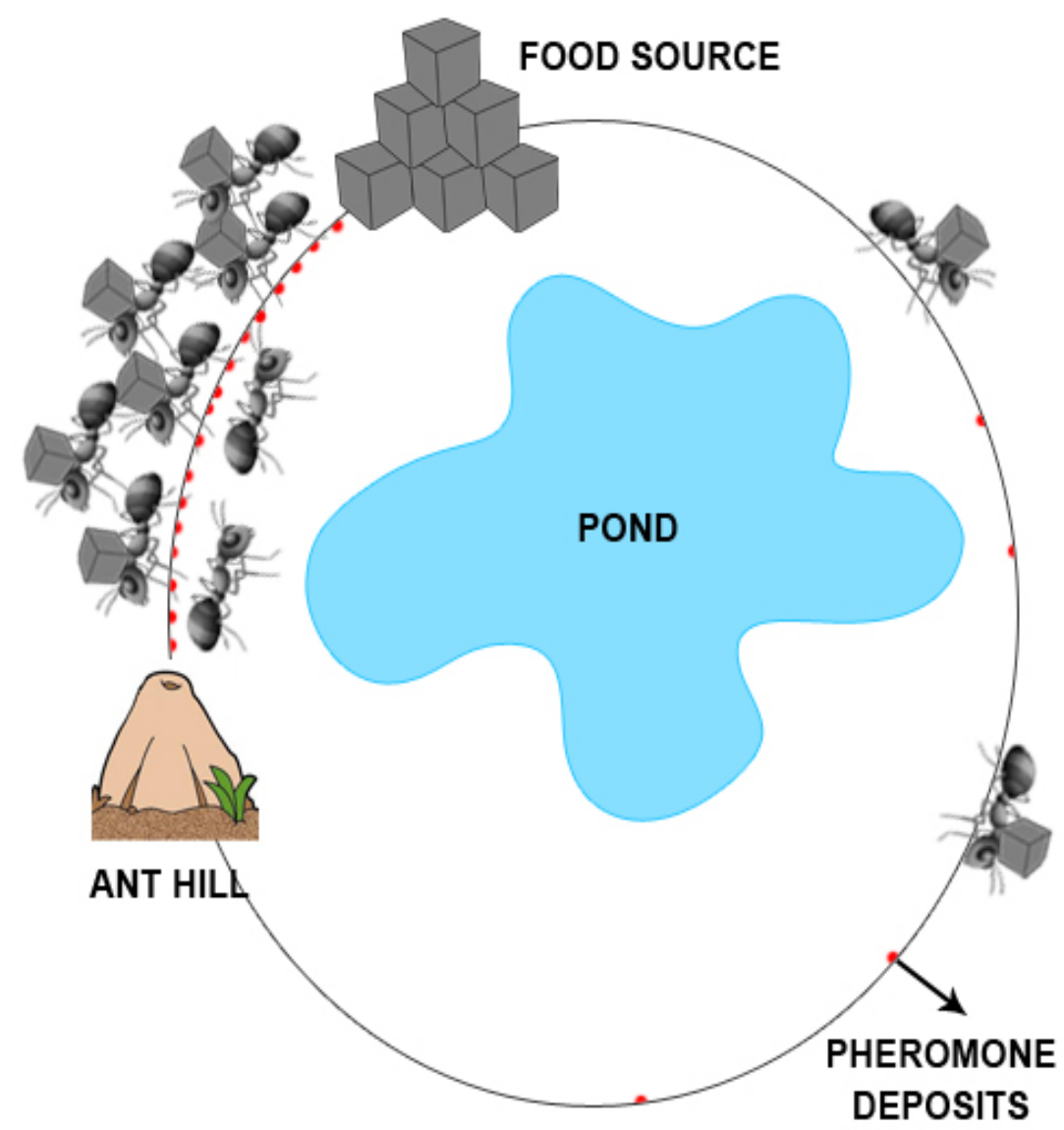

Fig -1: The pheromone trial on the longer path gets evaporated after some duration of time and the entire ant colony follows the shortest path.

ACO simulates the foraging behavior of the real ants in which they establish shortest path between the food source and the nest. While searching for the food source the ant randomly moves in the area surrounding its nest. While carrying the food source towards the nest they drop a chemical pheromone over the ground. Ants can smell pheromone and evaluate its quantity. Thus larger quantity of pheromone is concentrated on the shortest path and pheromone deposited on the longest path begins to evaporate. The path chosen by the ant contains large amount of pheromone concentration. Thus after acquiring the food source the ant leaves pheromone trial based on the quality and quantity of the food source. This pheromone trial helps other ants to find the shortest path for the food source. The indirect exchange of information through pheromone between the ants is known as stigmergy.

\section{ANT SYSTEM (AS)}

It was developed by Dorigo et al. in 1991 to solve the Travelling Salesman Problem (TSP). In the AS algorithm, the evaporation occurs all over the trail constructed by each ant. Besides that, pheromone is deposited along the trail proportionally to the quality of the corresponding solution. The pseudo code of the Ant System is as follows 
Ant System (AS)

1. for each ant colony

2. for each ant

3. Generate path

4. Evaluate path

5. evaporate pheromone in trails

6. deposit pheromone on trails

7. end for

8. end for

The ACO meta-heuristic consists of a colony of artificial ants with the characteristics to search good solutions to various optimization problems. Artificial ants have been inspired by the real ones although they can be enriched with some capabilities which are not found in real ants. Some similarities with real ants are the existence of a colony of cooperating individuals, pheromone trail and stigmergy, and the task of finding the shortest path between the nest and the food source. The main differences are: (i) artificial ants move from one discrete state to another, and (ii) they have an internal memory of the search procedure. Also artificial ants deposit an amount of pheromone which is a function of the quality of the solution found.

\section{HARMONY SEARCH ALGORITHM}

Harmony Search Algorithm (HSA) was developed by Zong Woo Geem et al. in 2001 and can be applied to solve many optimization problems like water distribution networks, ground water modeling, vehicle routing and many more. Harmony Search is music based metaheuristic optimization algorithm. When playing the music the orchestrator always tries establishing harmony by improvisation process. This harmony in the music is analogous to finding the optimality in an optimization process. The optimal solution to any problem corresponds to best solution with given constraints and parameters. The harmony depends on the aesthetics quality of music which is determined through pitch (frequency), timbre (sound quality) and amplitude (loudness) of musical instrument. Perfect harmony is achieved by adjusting the pitch which changes the frequency. In music theory the pitch $\mathrm{P}_{\mathrm{n}}$ is expressed using following formula

$$
P_{n}=69+12 \log _{2}(f / 440 H z)
$$

Zong Woo Geem et al. has introduced three methods for the process of optimization i.e. harmony memory, pitch adjusting and randomization. The harmony memory consists of random harmony solutions which are evaluated on the basis of accepting rate $r_{\text {accept }}$. Pitch adjustment is done by considering the pitch bandwidth $b_{\text {range }}$ and pitch adjusting rate $r_{p a}$. So we have following equation

$$
\mathrm{x}_{\text {new }}=\mathrm{x}_{\text {old }}+\mathrm{b}_{\text {range }}{ }^{*} \varepsilon
$$

where $\mathrm{x}_{\mathrm{old}}$ is the existing pitch value or solution from harmony memory and $x_{\text {new }}$ is new pitch after pitch adjusting step. Thus new solution is generated by adjusting the pitch which is better than existing solution. The pitch adjusting rate $r_{p a}$ controls the degree of adjustment. Higher value of $r_{p a}$ causes the solution to scatter around some potential optima.
Hence in many applications we use $r_{p a}=0.1 \sim 0.5$. Randomization deals with the diversity of solutions and helps to find the global optimal solution. The pseudo code of Harmony Search Algorithm is

\author{
begin \\ Objective function $\mathrm{f}(\mathbf{x}), \mathbf{x}=(\mathrm{x} 1, \mathrm{x} 2 \ldots \mathrm{xd})^{\mathrm{T}}$ \\ Generate initial harmonics (real number arrays) \\ Define $\left(r_{p a}\right)$, pitch limits and bandwidth \\ Define harmony memory accepting rate $\left(\mathrm{r}_{\text {accept }}\right)$ \\ while $(t<$ Max number of iterations ) \\ Generate new harmonics by accepting best harmonics \\ Adjust pitch to get new harmonics (solutions) \\ if(rand $>r_{\text {accept }}$ ), choose an existing harmonic randomly \\ else if $\left(\right.$ rand $\left.>r_{p a}\right)$, adjust the pitch randomly within limits \\ else generate new harmonics via randomization \\ end if \\ Accept the new harmonics (solutions) if better \\ end while \\ Find the current best solutions \\ end
}

\section{ARTIFICIAL BEE COLONY ALGORITHM}

Artificial Bee Colony (ABC) algorithm is swarm intelligence based optimization algorithm developed by Dervis Karaboga and colleagues in 2005. It mimics the intelligent foraging behavior of the honey bees. $\mathrm{ABC}$ can be used for optimizing a large set of numerical test functions. Three main components of the ABC algorithm are food sources, employed foragers and unemployed foragers. The food source is selected by the bee based on some properties like the amount of nectar, closeness to hive, taste of nectar and effort required for extracting. Employed forager is the bee which exploits the food source and provides other bees with rich set of information like distance, direction and profitability of the food source. Unemployed forager is the bee which searches for the food source. They are of two types i.e. scouts and onlookers. The scouts search the food source randomly while the onlookers search for the food source based on information provided by the employed forager. The exchange of the information takes place in the dancing area and the related dance is called as the waggle dance. There is higher probability of selecting the most profitable food sources by the onlooker bees. Let's consider two food sources A and B as shown in Figure2. Initially the unemployed bee starts searching for the food source without any previous knowledge and there are two cases. If the bee is scout it randomly searches for the food source. If the bee is onlooker it watches the waggle dance and selects the food source which has highest profitability. After acquiring the food source the bee becomes employed and starts unloading the nectar into its hive. After unloading the food the bee exhibits any one of the following behavior. 


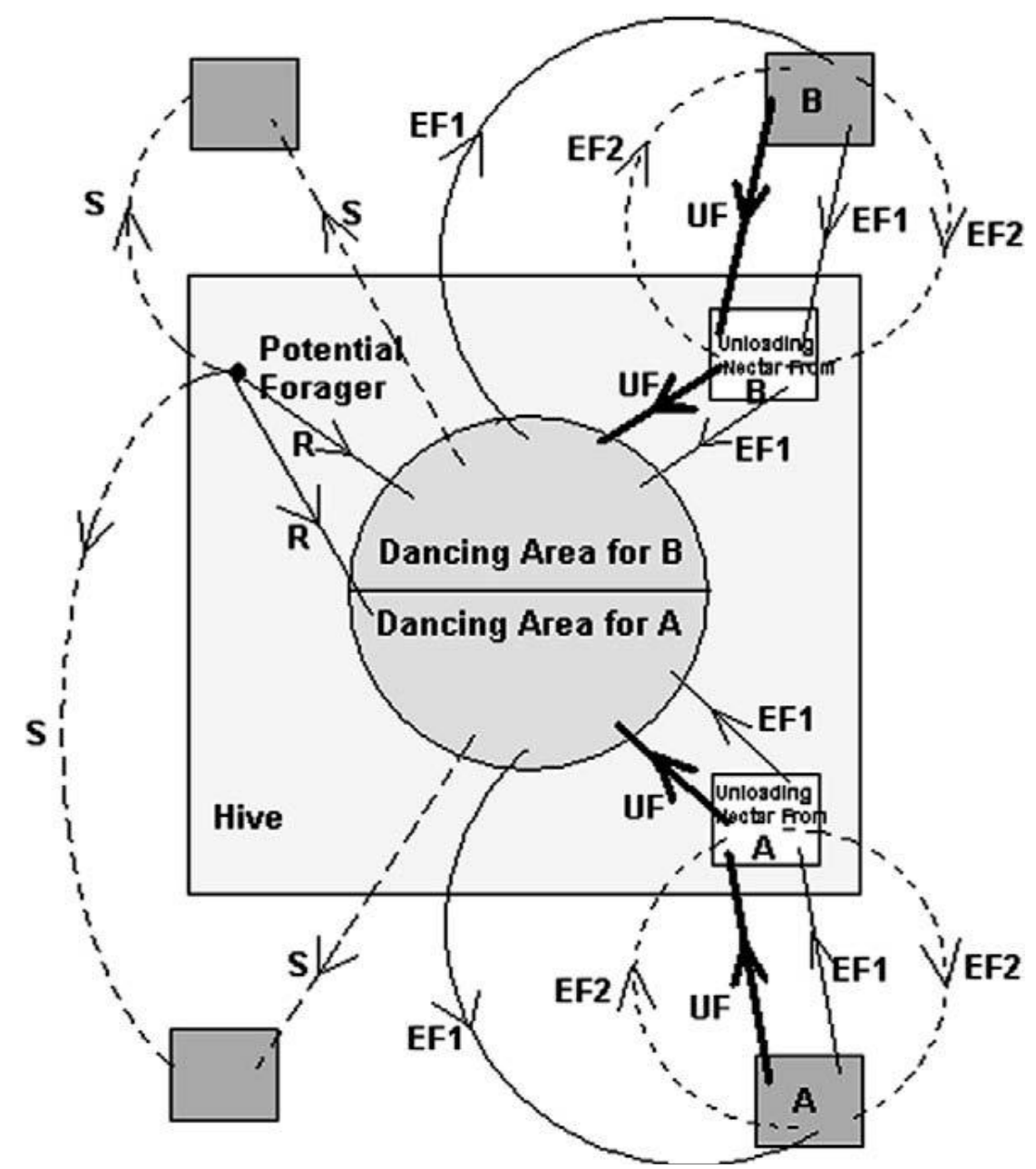

Fig -2: Foraging Behavior of Honey Bees.

1. The bee becomes Uncommitted Follower (UF) by not returning to the same food source again.

2. The bee returns to dancing area and exchanges the information to recruit some new bees before returning to the same food source (EF1).

3. The bee does not return to the dancing area or recruit new bees but continues to forage the same food source (EF2).

The main steps of the algorithm are as follows:

1. Population initialization

2. Repeat

3. Employed bees are placed on their food sources.

4. Onlooker bees are placed on food source based on the amount of nectar associated with a food source.

5. Scout bee searches randomly for the new food source.

6. The location of the best food source is memorized by bee.

7. Until the requirements are met

Initial population has same number of employed bees and onlooker bees and one scout bee. At the initialization stage the food sources are randomly selected by the bees and nectar quantity is evaluated. In ABC algorithm each cycle consists of three steps as follows.
- In first step the employed bees come into hive and share the information about the food sources in dancing area. The onlooker bee gets this information and searches the most profitable food source. The employed bee once again returns to the same food source because its location is memorized in the previous cycle. If the employed bee encounters a food source having higher nectar amount it memorizes this new location and forgets the old location.

- In second step the onlooker bees select the food source by evaluating the amount of nectar. As the nectar amount of the food source increases, the probability of that food source chosen also increases.

- In third step when the food source is abandoned it is replaced by the new food source determined by the scout bee.

\section{PARTICLE SWARM OPTIMIZATION}

It is evolutionary computation algorithm which was developed by Kennedy and Eberhert in 1995. It simulates the behavior of bird flocking together to seek the food source. While searching for the food source the birds communicate with each other and reach target within minimal duration of time. The population of PSO is 
initialized with random solutions called particles. Each particle has a capability to freely fly through the search space with some velocity which can be dynamically adjusted during search process. The original PSO algorithm is stated through following equation.

$$
\begin{gathered}
\mathrm{V}_{\mathrm{md}}=\mathrm{V}_{\mathrm{md}}+\mathrm{c1Rand1}()\left(\mathrm{P}_{\mathrm{md}}-\mathrm{X}_{\mathrm{md})}+\mathrm{c} 2 \operatorname{Rand} 2()\left(\mathrm{P}_{\mathrm{gd}^{-}}\right.\right. \\
\mathrm{X}_{\mathrm{md}) \ldots \ldots \ldots}(3) \\
\mathrm{X}_{\mathrm{md}}=\mathrm{X}_{\mathrm{md}}+\mathrm{V}_{\mathrm{md}} \ldots \ldots \ldots \ldots \ldots \ldots \ldots \ldots \ldots \ldots \ldots \ldots \ldots \ldots \ldots \ldots \ldots \ldots \ldots \ldots \ldots \ldots \ldots
\end{gathered}
$$

Where c1, c2 are positive constants and Rand1 () and Rand2 () generate random numbers between range 0 and $1 . \mathrm{X}_{\mathrm{ml}}$, $\mathrm{X}_{\mathrm{m} 2} \quad \ldots . . \mathrm{X}_{\mathrm{md}}$ represents the particles, $\mathrm{P}_{\mathrm{m} 1}, \mathrm{P}_{\mathrm{m} 2} \ldots . \mathrm{P}_{\mathrm{md}}$ represents the best fitness value of the particles. The index $g$ belongs to best particle among the population and $\mathrm{V}_{\mathrm{m} 1}, \mathrm{~V}_{\mathrm{m} 2}$ ..... $\mathrm{V}_{\mathrm{md}}$ represents the velocities of the particle. Equation (3) represents how the velocity is dynamically adjusted over the search process and Equation (4) represents how position is updated for each flying particle. If the value of $\mathrm{V}_{\mathrm{md}}$ exceeds user defined threshold Vmax then velocity of all particles is dynamically adjusted to Vmax. The pseudo code of the algorithm is given below

1. Initialize the particles in population with some random velocities and positions.

2. Repeat

3. For each particle calculate the best fitness value.

4. Compare the particles best fitness value with pbest. If current value is better than pbest then set pbest as best fitness value and change the location accordingly for this particle.

5. Index of particle with best fitness value among whole population is assigned to $\mathrm{g}$.

6. Dynamically change the velocity and position according to equation (1) and (2).

7. Until termination criterion is met.

\section{GENETIC ALGORITHM}

Genetic Algorithm was developed by Goldberg and inspired through Darwin's theory of Evolution. In this algorithm the initial population consists of chromosomes which represent solutions to the optimization problem. The fitness value is calculated for each chromosome which is composed of multiple genes. The chromosomes with higher fitness value are selected for crossover operation thereby producing offspring's. These offspring's have combination of parents genes. Also few chromosomes undergo mutation on genes. The number of chromosomes which will undergo crossover and mutation is controlled by crossover rate and mutation rate value.

Chromosome in the population that will remain for the next generation are selected based on Darwinian evolution rule. The chromosome which has higher fitness value will have greater probability of being selected again in the next generation. After several generations, the chromosome value will converge to a certain value which is the best solution for the problem.
The Algorithm

In the genetic algorithm process is as follows

1. Determine the number of chromosomes, generation, and mutation rate and crossover rate value

2. Initialize the population with Chromosome.

3. Process steps 4-8 until the number of generations is met

4. Evaluation of fitness value of chromosomes by calculating objective function

5. Chromosomes selection

6. Crossover operator

7. Mutation operator

8. Produce Offspring

9. Chromosome with higher fitness value is best solution.

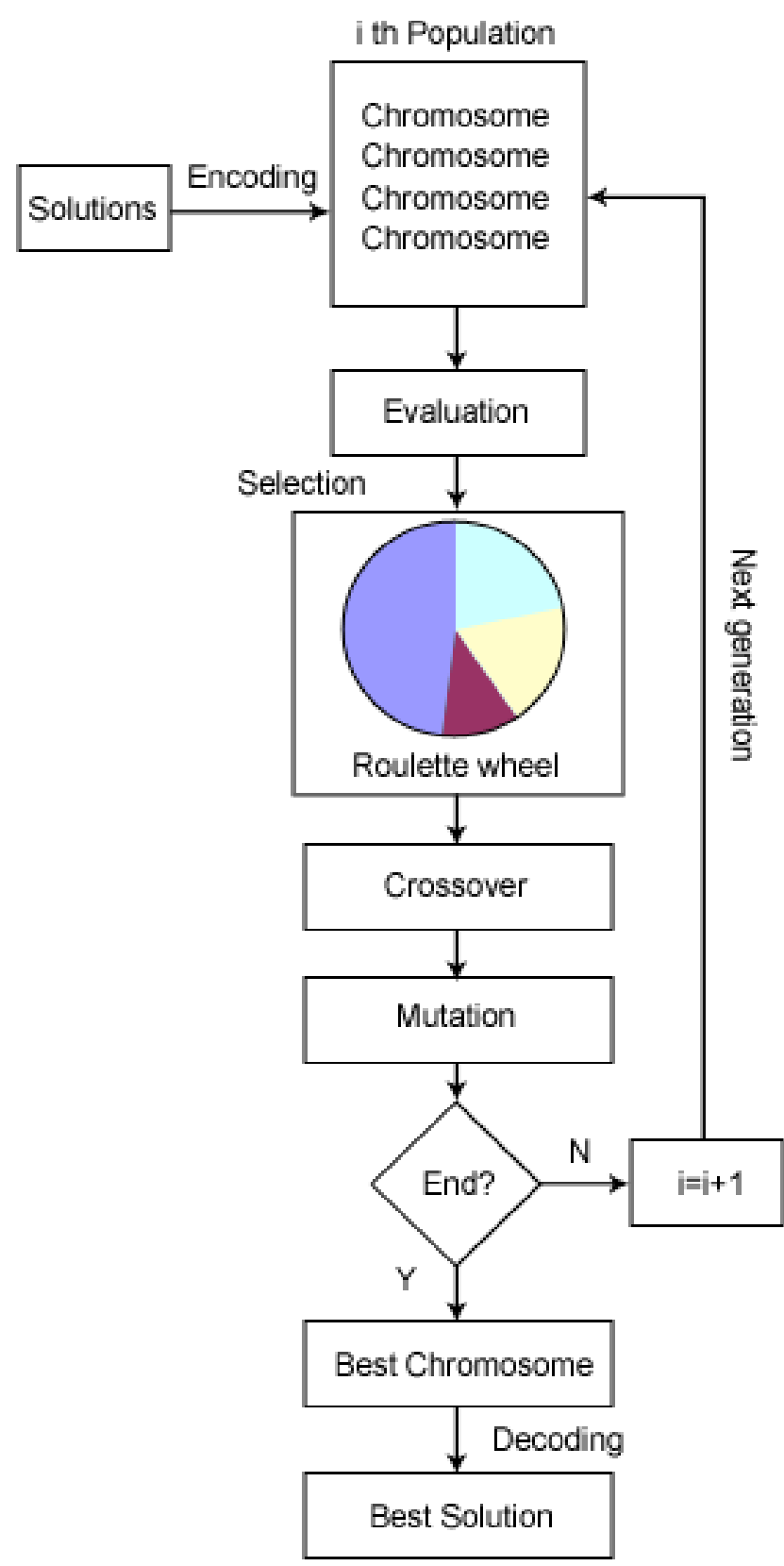

Fig -3: Flowchart of Genetic Algorithm. 


\section{CONCLUSION}

In this paper we have reviewed many optimization techniques which have been used for image compression. In spite of existence of various image compression algorithms optimization techniques are widely used by many researchers to get best compressed image with minimal loss of information. One important advantage of using the optimization technique is that it reduces the time of compression and also helps to increase the compression ratio without degrading the quality of the image.

\section{REFERENCES}

[1] Shu-Chuan Chu, Hsiang-Cheh Huang, John F. Roddick, and Jeng-Shyang Pan - Overview of Algorithms for Swarm Intelligence, International Conference on Computer and Computational Intelligence(ICCCI 2011), Part I, LNCS 6922, pp. 28 41, 2011. (C) Springer-Verlag Berlin Heidelberg 2011.

[2] R.C Eberhart, Y. Shi, J. Kennedy- Swarm Intelligence, Morgan Kaufmann, 2001.

[3] K.Uma, P.Geetha, A.Kannan, K.Umanath - Image Compression Using Optimization Techniques, eISSN: 2278-067X, p-ISSN: 2278-800X, Volume 5, Issue 5 (December 2012), PP. 01-07, www.ijerd.com

[4] Mrs. HarshaD.Zope, Prof. Mrs.Pallavi Y. Patil Comparative Study of Different Approaches of Image Compression,(ISSN 2250-2459, Volume 2, Issue 1, January 2012), Website: www.ijetae.com .

[5] DervisKaraboga, BahriyeAkay- A comparative study of Artificial Bee Colony algorithm, Applied Mathematics and Computation, Volume 214, 2009, pp 108-132, Website www.elsevier.com/locate/amc.

[6] Ryan Rey M. Daga, John Paul T. Yusiong- Image compression using Harmony Search Algorithm, International Journal of Computer Sciences, Vol 9, Issue 5, No 3, 2012, www.IJCSI.org.

[7] Sarang Bansod, Sweta Jain- Improved Harmony Search Algorithm for Color Image Compression, International Journal on Recent and Innovation Trends in Computing and Communication, Volume 2, Issue 6, June 2014.

[8] Z. W. Geem- Music inspired Harmony Search Algorithm Theory and Applications, Studies on Computational Intelligence, Vol 199, 2009, pp. 213.

[9] Simranjeet Kaur, Prateek Agarwal, Rajbir Singh Rana - Ant Colony Optimization : A Technique used for Image Processing, IJCST Vol. 2, Issue 2, June 2011.

[10] Christian Blum- Ant Colony OptimizationIntroduction and Recent Trends, Physics of Life Reviews, Vol2,2005, pp 353-373, www.elsevier.com/locate/amc.

[11] C. Martinez- An ACO Algorithm for Image Compression, CLEI Electronic Journal, Vol 9, No 2, 2006, pp. 2-5.
[12] M. Mohamed Ismail, Dr.K.Baskaran - Hybrid Lifting Based Image Compression Scheme Using Particle Swarm Optimization Algorithm And Artifical Bee Colony Algorithm,International Journal of Advanced Research in Computer and Communication Engineering Vol. 3, Issue 1, January 2014.

[13] M. Mohamed Ismail, Dr. K. Baskaran - Adaptive Lifting Based Image Compression Scheme Using Artificial Bee Colony Algorithm, International Journal of Electronics Communication and Computer Engineering, Volume 4, Issue 1, ISSN (Online): 2249-071X, ISSN (Print): 2278-4209.

[14] S.K Mitra, C. A Murthy, M.K. Kundu- Technique for fractal image compression using Genetic Algorithm, IEEE Trans, Image Processsing, Vol 4, No. 7, pp. 586-593, 2006.

[15] Chen Shuwang An tao Hao litao, IEEE 2009, DCT based image compression using Genetic Algorithm.

\section{BIOGRAPHIES}

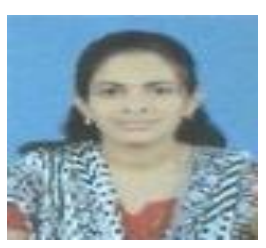

Shet Reshma Prakash received B.E in Information Technology from Mumbai University and currently pursuing $\mathrm{M}$ Tech in Computer Science from VTU, Belgaum. Field of interest includes image processing.

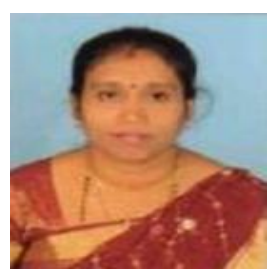

Vrinda Shiva Shetty received B.E from Gulbarga University and M.Tech degree from VTU in Computer Science and Engineering and presently pursuing Ph.D. in Image Compression from the Gulbarga University Gulbarga, Karnataka. Field of Interest includes Image Processing, Evolutionary Computation. 